\title{
Optimal joint routing and link scheduling for real- time traffic in TDMA Wireless Mesh Networks
}

\author{
Paola Cappanera \\ Dipartimento di Sistemi e Informatica \\ University of Florence, Italy \\ paola.cappanera@unifi.it
}

\author{
Luciano Lenzini, Alessandro Lori, Giovanni Stea, Gigliola Vaglini \\ Dipartimento di Ingegneria dell'Informazione \\ University of Pisa, Italy \\ \{1.lenzini, a.lori, g.stea, g.vaglini\}@iet.unipi.it
}

\begin{abstract}
We investigate the problem of joint routing and link scheduling in Time-Division Multiple Access (TDMA) Wireless Mesh Networks (WMNs) carrying real-time traffic. We propose a framework that always computes a feasible solution (i.e. a set of paths and link activations) if there exists one, by optimally solving a mixed integer-non linear problem. Such solution can be computed in minutes or tens thereof for e.g. grids of up to $4 \times 4$ nodes. We also propose heuristics based on Lagrangian decomposition to compute suboptimal solutions considerably faster and/or for larger WMNs, up to about 50 nodes. We show that the heuristic solutions are near-optimal, and we exploit them to investigate the optimal placement of one or more gateways from a delay bound perspective.
\end{abstract}

Keywords-Link Scheduling; Routing; Wireless Mesh Networks; Real-time Traffic; Worst-Case Delay

\section{INTRODUCTION}

Wireless Mesh Networks (WMNs) [1] are a cost-effective technology for providing broadband access at the edge of wireline networks, or in remote, rural, or difficult-to-wire areas. Interference among wireless links with overlapping coverage can be sorted out in either the frequency or the time domain. In the first case, different channels are assigned to interfering links, a problem known as channel assignment In the second case, which is the one dealt with in this paper, the full frequency spectrum is given to each link, but interfering links are activated on a Time Division Multiple Access (TDMA [2]) basis. In this case, time is slotted and synchronized, and a link scheduling algorithm activates only sets of non-interfering links in the same time slot. Link scheduling algorithms are generally more effective if they take into account the (known or estimated) traffic demand and link scheduling is considered jointly with routing, WMNs generally requiring multi-hop communications. Cross-layer approaches where link scheduling or channel assignment and routing are jointly addressed have been extensively studied [3]-[7] in the past few years.

In the recent past, a growing number of works have envisaged using WMNs for transmitting real-time traffic, e.g. road traffic information [34], video surveillance [35], etc. Real-time traffic requires a priori end-to-end delay bounds to be guaranteed by the underlying WMN. However, comparatively few works so far have taken into account the problem of computing link schedules subject to arbitrary delay bounds, either given a pre-specified routing plan or jointly with routing. Some (e.g.,
[24]-[26]) tackle the problem of minimizing the TDMA delay, i.e. the sum of the waiting times due to multi-hop TDMA scheduling. Some works ([15]-[18]) aim at guaranteeing a minimum rate, which guarantees that the maximum delay is finite. Others, finally, aim at optimizing the throughput [19][22], or reducing the average delay [33]. While all the above goals are indeed important and worth pursuing, they are not enough to guarantee that pre-specified worst-case delays are enforced if it is actually possible to do so. For instance, we showed in [9] that minimizing the TDMA delay, as done in [26], yields schedules that largely violate pre-specified delay bounds, even though it is possible to find alternative, delayfeasible schedules. Our previous works [8]-[10] are actually the first to consider delay-constrained link scheduling in WMNs, also evaluating different architectural options for flow aggregation. However, routing is left outside the scope of these works, by assuming either a tree network topology, with a single possible path from each node to the network gateway ([8], [10]), or an arbitrary but given routing plan, upon which a delay-feasible link schedule is computed ([9]). Tackling the problem of routing and link scheduling separately (e.g., in a cascading approach) leads to a loss in effectiveness. In fact, in a WMN, interfering links cannot be active simultaneously: as a consequence, the rate along routes selected without taking into account link scheduling is unpredictable. As a consequence, sets of flows may unnecessarily be declared unschedulable.

In this paper we investigate the problem of joint routing and link scheduling of leaky-bucket constrained flows that request worst-case delay guarantees. We formulate it as an optimization problem, the Delay-Aware Routing and Scheduling (DARS) problem, with the objective of minimizing the maximum deadline violation. When a solution with a negative objective is computed, each flow will follow a route that makes it meet its deadline despite interference. We show that the problem can be optimally solved for networks of up to few nodes (e.g., a $4 \times 4$ grid). To allow for larger scales, we propose two suboptimal heuristics, that rely on extrapolating the link conflict serialization (LCS) from the DARS. In the LCS, sequences of conflicting link activations are statically precomputed using a coloring approach [32], so as to minimize the longest sequence. In the remaining reduced DARS, the activation of each link is computed jointly with routing, so as to minimize the maximum deadline violation. Once conflicting links are serialized, the reduced DARS problem can be solved optimally for a larger scale (e.g., a $5 \times 5$ grid); beyond that scale, optimality has to be traded off for computation time. For this reason, we propose a 
faster scheme based on a Lagrangian decomposition of the reduced DARS. We show that this heuristic scheme is considerably faster (which allows larger-scale WMNs to be analyzed) and performs close to the optimum. Furthermore, this model can be used to extract useful information related to a WMN, e.g. where to place an Internet gateway node, and whether and when it is profitable to have more than one such node.

The rest of the paper is organized as follows: Section II reports the system model and the problem formulation. In Section III we discuss the properties of the optimal solution and present heuristics. We report performance evaluation results in Section IV, and discuss the related work in more detail in Section V. Section VI concludes the paper.

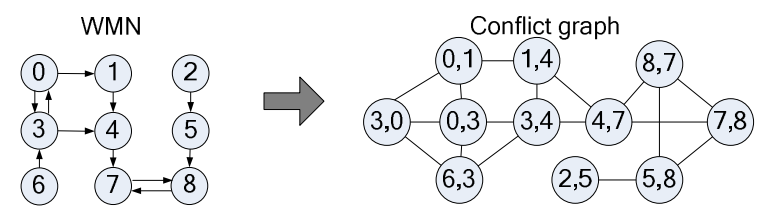

Figure 1. Logical connectivity graph (left) and conflict graph (right) of a WMN.

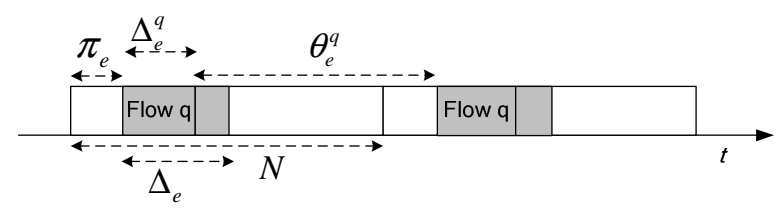

Figure 2. Relevant quantities in link scheduling.

\section{SYSTEM MODEL AND PROBLEM FORMULATION}

The framework developed in this paper relies on basic Network Calculus concepts, i.e. arrival curve, service curve and delay bound. Interested readers can find background in [11], from which we also borrow notation.

We assume that each mesh router is equipped with a single time-slotted channel. Transmission slots of a fixed duration $T_{s}$ are grouped into a frame of $N$ slots, which is periodically repeated every $N \cdot T_{S}$ time units. For instance, in 802.16 networks the frame length is usually set to $5 \mathrm{~ms}$. Each slot is assigned to a set of non-interfering links through conflict-free link scheduling. At every slot, a subset of links may be activated for transmission only if no conflicts occur at the intended receivers. The WMN is modeled through a connectivity graph, $G=(V, E)$, whose nodes $V=\left\{v_{1}, \cdots, v_{n}\right\}$ are mesh routers and whose edges $E=\left\{e_{1}, \cdots, e_{m}\right\}$ are directed links connecting nodes within transmission range. We assume that each link $e$ has a constant transmission rate $W_{e}$.

Nodes are traversed by flows (i.e., distinguishable streams of traffic). Let $Q$ denote the set of all flows. Flow $q \in Q$ is to be routed through a path $P_{q} \subseteq E$ between its source $s(q)$ and destination $d(q)$. Each flow has a delay constraint, specified as an end-to-end delay bound or deadline $\delta_{q}$. At the ingress node, its arrivals are constrained by a leaky-bucket shaper, with a burst $\sigma_{q}$ and a rate $\rho_{q}$. Packets of each flow are buffered separately at each link. The purpose of this paper is to describe a joint routing and link scheduling scheme that computes a conflict-free schedule which does not violate the required delay bounds whenever it is possible to do so. We first identify the constraints that ensure the conflict-free property, and then move to describing those related to delay feasibility.

The physical interference phenomenon is modeled by means of the widely used protocol interference models ([4], [12]). For each edge of the network $e \in E$ we define a conflicting set of edges $\mathcal{I}(e)$ which includes all the edges belonging to $E$ which interfere with $e$ ( $\mathcal{I}(e)$ contains $e$ itself); the interference condition is straightforwardly defined as follows:

$$
\sum_{i \in \mathcal{I}(e)} x_{i}(t) \leq 1 \text {, if } e \text { is active in slot } t=1,2, \ldots, N,
$$

where $x_{e}(t)$ is a binary variable, such that $x_{e}(t)=1$ if link $e \in E$ is active in slot $t$, and 0 otherwise. This means that, if edge $e$ is active in slot $t$, the associated interfering set $\mathcal{I}(e)$ must contain one active edge only (which is the edge $e$ itself). We translate the interference condition to a conflict graph $G_{c}=(E, C)$, shown in Figure 1, whose nodes are the set of links of the logical connectivity graph and whose edges $C=\left\{c_{1}, \ldots, c_{r}\right\}$ model the conflicts within the network.

Half-duplex constraints are implicitly accounted for into the interference constraints, links being unidirectional. Hence a set $\mathcal{I}(e)$ can be easily obtained by retrieving the one-hop neighborhood of $e$ in the conflict graph, e.g. for Figure 1 we have $\mathcal{I}(7,8)=\{(4,7),(5,8),(8,7)\}$. Given a conflict graph $C$, only conflicts between active links, i.e. those with a nonnull flow, have to be considered. We thus define $C_{f} \subseteq C$ as the subset of conflicts involving active links:

$$
C_{f}:=\left\{(i, j) \in C: f_{i}>0 \text { and } f_{j}>0\right\},
$$

where $f_{i}$ denotes the flow going trough link $i$.

Following the notation in [8]-[10], we define an activation offset $\pi_{e}$ for link $e, 0 \leq \pi_{e} \leq N$, and its transmission duration $\Delta_{e}$. Since time is slotted, both are non negative integers. Figure 2 shows the above quantities, plus others that will be defined in the following. The assumption that one (instead of several) activation of a link in a frame is allowed stems from the fact that, in several technologies (e.g., WiMAX) the link scheduling map is communicated to the various nodes of a WMN in-band: in this case, the shorter the map is, the smaller the overhead is.

The schedule must ensure the conflict-free condition: while a link is transmitting, all conflicting links must refrain from transmitting. For any pair of links $i$ and $j$ which are neighboring nodes in $C_{f}$ we have:

- if $j$ transmits after $i$, it must wait for $i$ to complete the transmission, i.e. $\pi_{i}-\pi_{j}+\Delta_{i} \leq 0$.

- Otherwise, the symmetric inequality holds, i.e. $\pi_{j}-\pi_{i}+\Delta_{j} \leq 0$

In order to linearize the combination of the above constraints, we introduce a binary variable $o_{i j},(i, j) \in C_{f}$, which is 1 if $i$ transmits after $j, 0$ otherwise. The left-hand side of the previous constraints can thus be upper bounded by $N$ regardless of the relative transmission order, as $\pi_{i}$ and $\Delta_{i}$ belong to $[0, N]$. This completes the formulation of the conflictfree constraints, which are necessary and sufficient conditions:

$$
\begin{array}{ll}
\pi_{i}-\pi_{j}+\Delta_{i} \leq N \cdot o_{i j} & \forall(i, j) \in C_{f} \\
\pi_{j}-\pi_{i}+\Delta_{j} \leq N \cdot\left(1-o_{i j}\right) & \forall(i, j) \in C_{f}
\end{array}
$$

For a schedule to be valid, each link must also complete its transmission within the frame duration, i.e.:

$$
\pi_{i}+\Delta_{i} \leq N \quad \forall i \in E .
$$


Additional constraints are needed to keep into account the end-to-end delay requirements. During its activation, each link $e$ transmits traffic of all the flows that traverse that link. We can therefore partition the link's $\Delta_{e}$ among them, i.e. $\Delta_{e}=\sum_{q: e \in P} \Delta_{e}^{q} . \Delta_{e}^{q}$ is the link activation quota reserved for flow $q$, which needs not be an integer, since when a link $e$ is activated it can switch among backlogged queues regardless of slot boundaries. We assume that backlogged flows traversing $e$ are served in the same (arbitrary) local order, and we call $I_{e}$ the ordered set of the flow indexes. We assume that each backlogged flow $q$ is served for no less than $\Delta_{e}^{q}$. If a flow is idle, its service time can be exploited by other backlogged flows at $e$, as long as the transmission from any flow $z$ starts within at most $\sum_{x \in I \cdot x<z} \Delta_{e}^{x}$ from $\pi_{e}$. Therefore, flow $q$ has a guaranteed rate at link $e$ equal to:

$$
R_{e}^{q}=W_{e} \cdot \Delta_{e}^{q} / N .
$$

Since each flow transmits once per frame, a maximum inter-service time is guaranteed for that flow, and it is equal to:

$$
\theta_{e}^{q}=\left(N-\Delta_{e}^{q}\right) \cdot T_{S},
$$

irrespective of the local ordering at each link. Therefore, each link of a mesh router is a rate-latency server [11] for the flows traversing it, with a rate $R_{e}^{q}$ and a latency $\theta_{e}^{q}$. Accordingly, each flow has an end-to-end delay bound equal to (see [11]):

$$
D_{q}=\left\{\begin{array}{cc}
\sum_{e \in P_{q}} \theta_{e}^{q}+\sigma_{q} / R_{\min }^{q} & \text { if } \rho_{q} \leq R_{\min }^{q} \\
\infty & \text { otherwise }
\end{array},\right.
$$

where $R_{\min }^{q}=\min _{e \in P_{q}}\left\{R_{e}^{q}\right\}$. The first addendum in (5) is called latency delay, and it is due to link scheduling and arbitration of the flows at the links. The second is called burst delay, and it is the time it takes for the flow's burst to be cleared at the minimum guaranteed rate.

Given the traffic, the network topology and the conflict graph, our purpose is to find a joint conflict-free routing and scheduling which is also feasible from a delay point of view. To achieve this, we formulate the Delay-Aware Routing and Scheduling (DARS) problem as follows:

$$
\begin{aligned}
& \min V_{\max } \\
& \text { s.t. : } \quad \sum_{e \in E} \theta_{e}^{q}+\frac{\sigma_{q}}{R_{\min }^{q}}-\delta_{q} \leq V_{\max } \\
& \forall q \in Q \\
& \left(N-\Delta_{e}^{q}\right) \cdot T_{S} \leq \theta_{e}^{q}+N \cdot T_{S} \cdot\left(1-t_{e}^{q}\right) \\
& R_{\min }^{q} \leq \frac{W_{e}}{N} \cdot \Delta_{e}^{q}+\left(1-t_{e}^{q}\right) \cdot \max _{i \in E}\left\{W_{i}\right\} \\
& \forall e \in E, \forall q \in Q \\
& \forall e \in E, \forall q \in Q \\
& \forall e \in E, \forall q \in Q \\
& \forall e \in E, \forall q \in Q \\
& \forall e \in E \\
& \forall e \in E \\
& \forall i, j \in C_{f} \\
& \forall i, j \in C_{f} \\
& \forall i \in E \\
& \pi_{i}+\Delta_{i} \leq N \\
& \sum_{e \in O U T(v)} t_{e}^{q}-\sum_{e \in I N(v)} t_{e}^{q}=\left\{\begin{array}{cc}
1 & \text { if } v=s(q) \\
-1 & \text { if } v=d(q) \\
0 & \text { otherwise }
\end{array}\right. \\
& \forall v \in V, \forall q \in Q \\
& t_{e}^{q}, o_{i j} \in\{0,1\}, R_{\min }^{q}, \theta_{e}^{q}, \Delta_{e}^{q} \geq 0, \Delta_{e}, \pi_{e} \in Z_{0}^{+} \\
& \forall e \in E, \forall q \in Q
\end{aligned}
$$

The objective function to be minimized is the maximum $d e$ lay violation $V_{\max }$, defined as $V_{\max }=\max _{q \in Q}\left\{D_{q}-\delta_{q}\right\}$. If the optimum is negative, then the DARS problem has a solution which is feasible from a delay point of view. There are two sets of variables, related to link scheduling $\left(o_{i j}, \pi_{e}, \Delta_{e}\right)$ and routing $\left(t_{e}^{q}\right)$ decisions. As for routing, $t_{e}^{q}=1$ iff. flow $q$ traverses link $e$. As single-path (as opposed to multipath) routing is assumed, $t_{e}^{q}$ are binary. Constraints $(x i)$ ensure flow conservation at each node. Constraints $(i$-vii) ensure a delay-aware link scheduling. Specifically, $(i)$ represents $D_{q}-\delta_{q}$ according to (5) for flow $q$, assuming that its delay is finite. Constrains (ii-iv) include at the right hand side terms which depend on $\left(1-t_{e}^{q}\right)$ and $t_{e}^{q}$. Those terms are computed such that, if $t_{e}^{q}=0$, then the constraints always hold regardless of the value given to $\Delta_{e}^{q}, \theta_{e}^{q}, R_{\min }^{q}$. In other words, those constraints are inactive for those links that are not traversed by a flow. On the other hand, when $t_{e}^{q}=1$, (ii) sets the latency according to (4), (iii) guarantees that $R_{\min }^{q}$ is the minimum guaranteed rate among all the links traversed by flow $q$, i.e. $R_{\min }^{q}=\min _{e: t^{q}=1}\left\{W_{e} \cdot \Delta_{e}^{q} / N\right\}$, and (iv) ensures that the activation quota for flow $q$ is set according to (3), thus ensuring that the delay is finite. On the other hand, constraints $(v)$ and (vi) are active when $t_{e}^{q}=0$, when they guarantee that $\Delta_{e}^{q}$ is forced to zero when flow $q$ does not traverse link $e$. Those constraints always hold when $t_{e}^{q}=1$, instead. Constraint (vii) relates the activation of a link with the activation quotas of each flow traversing it. Constraints (viii-x) mirror (1)-(2), and are thus related to conflict-free scheduling.

Note that, since the routing is specified as part of the model, the latter allows one to account for both local traffic, directed from one node to another, and Internet traffic, directed from/to an Internet gateway node (i.e., both uplink and downlink). Furthermore, if the WMN has more than one gateway node, a straightforward modification of the model allows one to perform gateway selection, i.e. to select the gateway through which each flow has to be routed to guarantees the best objective. As shown in Figure 3, all it takes is to add a virtual supergateway node, connected solely to the gateways via mutually non-interfering links of suitable capacity (e.g., T1 or higher), and to select the latter as the source/destination node for all the Internet traffic.

The DARS problem is a Mixed Integer Non-Linear (MINLP) problem, whose non-linear constraints are convex and for which efficient general purpose MINLP solver (e.g. [13],[14]) exist. The latter can be easily re-formulated as a quadratic problem by introducing auxiliary variables, which makes it possible to use the efficient solver CPLEX [13]. Despite the quadratic formulation, the solution time of the above problem is prohibitive for mesh networks of medium to large size. For instance, CPLEX may take days to find the optimum for a $4 \times 4$ grid, and cannot solve a $5 \times 5$. For this reason, in the next section we present a heuristic approach to solve the DARS problem.

Before moving to the heuristics for the DARS, we justify the need to solve the routing and link scheduling jointly via a simple example. Figure 4 reports a sample $4 \times 4$ grid mesh, where four homogeneous flows need be routed from their source (nodes 0-3) to the gateway (node 15). It is $\sigma=1000$, $\rho=2000, \delta=30$ for all flows. The link capacity is 9600 for all links except $(7,11)$, whose capacity is 5000 . The figure also reports the routes selected by the DARS (the other variables are 
omitted for ease of reading). A quick glance suffices to convince the reader that these routes are not shortest paths, and it takes only a little more to verify that no shortest-path routing leads to a feasible link scheduling. For instance, if flow 3 were routed along its shortest path 3-7-11-15, link $(7,11)$ should be active for at least $40 \%$ of the time, leaving no more than $60 \%$ for conflicting link 11-15, which would then be unable to support flows 1, 2, 3 together. The latter, in fact, require an activation of $62.5 \%$ on that very link just to keep their delay bounded, let alone below any pre-specified requirement.

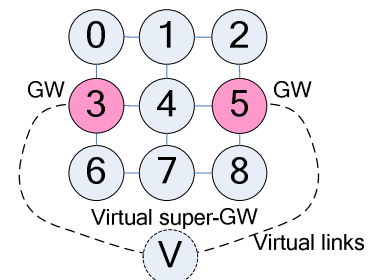

Figure 3. Logical topology of a WMN with more than one gateway and gateway selection.

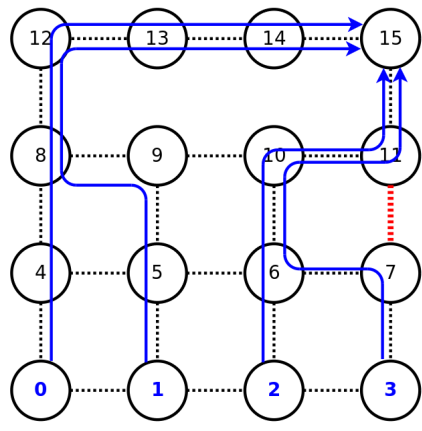

Figure 4. Sample mesh

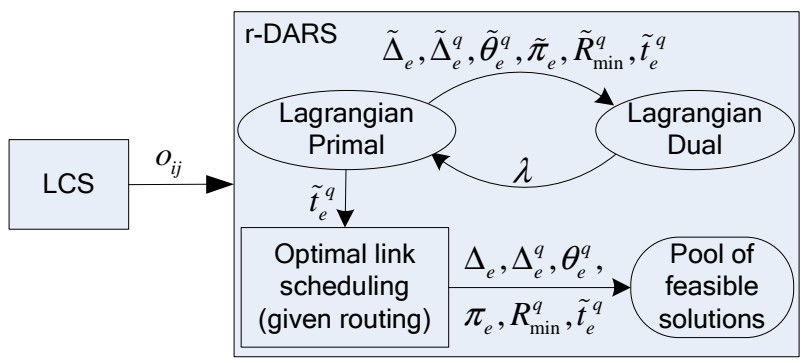

Figure 5. Separate heuristic approach

\section{HEURISTIC SOLUTIONS}

The high complexity of the DARS problem stems from the high number of binary variables related to conflict $\left(o_{i j}\right)$ and routing $\left(t_{e}^{q}\right)$. Of course, we cannot separate the routing variables without incurring in the problems outlined in the previous example. Therefore, in order to reduce the computation time, we separate the link conflict serialization ( $L C S$ ) from the DARS problem. In other words, we set the $o_{i j}$ variables offline, based on the conflict graph, and then solve the reduced DARS, where the $o_{i j}$ are constants. As we will show later on, this allows larger-scale problems to be solved, with a negligible loss of accuracy. To increase the scale further, we also propose a Lagrangian heuristic to solve the reduced DARS (r-DARS henceforth) problem suboptimally. We first describe how to solve the LCS, and then we move to the r-DARS. Our solution scheme is detailed in Figure 5.

A.

Link Conflict Serialization

Solving the LCS problem consists in setting the $o_{i j}$ variables, i.e. directing the edges in the conflict graph, which in turn translates to serializing conflicting links within the frame. In fact, all the links belonging in the same clique in the conflict graph - e.g., $(0,1),(1,4)$ in Figure 1 - cannot be activated in parallel, hence have to be serialized. For instance, assuming one-hop interference, a link may belong to up to two cliques (i.e., those of either ends). For instance, $(0,1)$ also belongs to a 3 -clique with $(3,0)$ and $(0,3)$, and to a 2 -clique with $(1,4)$. We remark that one-hop interference is not a mandatory assumption in our model. The objective to be pursued by the LCS is thus to minimize the maximum length path in the resulting directed conflict graph, i.e. to minimize the max number of serialized links. This can be done by employing a general Kcoloring method [32]. The K-coloring is exponential in the number of vertices. However, it can be solved up to scales much larger than the ones we are dealing with, and efficient methods - e.g., based on column generation [36] - can be exploited to solve the problem at larger scales.

Thus the LCS can be solved optimally, given the conflict graph. Therefore, as traffic changes, a new routing and link scheduling can be computed without modifying the conflict serialization. The negative side of solving the LCS without taking traffic into account is that a possibly short path in the conflict graph (i.e., one with few links) may end up carrying a large amount of traffic because of routing, and hence become critical. Nevertheless, since routing decisions are taken afterwards in the r-DARS, flows would be routed around such critical paths as a consequence of routing decisions.

$B$.

Lagrangian heuristic

The r-DARS is still a complex problem. While it can be solved in a matter of seconds in a $4 \times 4$ grid, it takes hours to solve it on a $5 \times 5$ grid. Therefore, we propose a heuristic scheme to solve it. The latter is based on a Lagrangian relaxation, which has a twofold advantage: (i) by exploiting the very structure of the r-DARS, it allows the problem to be partitioned, hence gaining in efficiency and/or scale; (ii) it allows one to compute both a lower and an upper bound on the optimum solution to the r-DARS. We first explain how to obtain a Lagrangian relaxation, and then show how the heuristic is built upon the latter.

The r-DARS has two blocks of variables: the link scheduling variables, involved in constraints $(i),(v i i i-x)$ and the routing variables in constraint (xi). In addition, a set of coupling constraints, i.e. (ii-vi), collate link scheduling and routing decisions. In the absence of the latter, r-DARS could be decomposed in two subproblems: a link scheduling problem and a routing problem respectively. Hence we perform a Lagrangian relaxation with respect to the coupling constraints: the latter are dualized by inserting them in the objective function and associating a non-negative Lagrangian multiplier $\lambda_{i}$ to each of them. The Lagrangian primal problem to be solved is the following: 


$$
\begin{aligned}
\varphi(\lambda) & =\min _{\text {s.t. }(i),(v i i-x)}\left\{V_{\max }+s\left(\lambda ; \Delta_{e}, \Delta_{e}^{q}, \theta_{e}^{q}, \pi_{e}, R_{\min }^{q}\right)\right\} \\
& +\min _{\text {s.t. }(x i)}\left\{r\left(\lambda ; t_{e}^{q}\right)\right\}
\end{aligned}
$$

where $s\left(\lambda ; \Delta_{e}, \Delta_{e}^{q}, \theta_{e}^{q}, \pi_{e}, R_{\min }^{q}\right)$ and $r\left(\lambda ; t_{e}^{q}\right)$ are linear cost functions depending on the Lagrangian multipliers (updated Lagrangian costs). The Lagrangian multiplier $\lambda_{i}$ plays two roles: i) it penalizes the variables for which the relaxed $i$-th constraint is violated by adding a positive term to the original objective function, and ii) it favors solutions for which the relaxed $i$-th constraint is satisfied, by adding a negative term to the objective function. Function $\varphi()$ is separable: for a given value of $\lambda$, solving the Lagrangian primal implies solving separately a scheduling problem and a routing problem, which is considerably faster than solving them jointly. Yet this scheme keeps routing and scheduling together through the multipliers, hence retaining the benefits of a joint approach. The solution thus computed is a lower bound on the optimum of the r-DARS, and is in general infeasible. It is thus necessary to compute the best lower bound among the possible choices of $\lambda$, i.e., to solve the Lagrangian dual:

$$
\max _{\lambda \geq 0}\{\varphi(\lambda)\} .
$$

By iterating between the primal and dual problems (see Figure 5), the solution moves towards the admissible solution from the outside. However, this scheme may never converge to an admissible solution. For this reason, we choose to exploit the routing part of the solution of the Lagrangian primal (i.e., the $t_{e}^{q}$ variables), and we solve the reduction of the r-DARS where routing variables are set (optimal link scheduling in Figure 5). This last box entails solving a Mixed Integer NonLinear problem, whose non-linear constraints are convex. If a feasible link scheduling is computed on a given routing, then the solution verifies all the constraints, and is thus admissible for the r-DARS problem (although not necessarily optimal), hence it is an upper bound on the optimum. As the Lagrangian scheme is iterated, possibly many feasible solutions are computed this way and stored in a pool. When the Lagrangian converges:

a) the best solution in the pool is returned.

b) the lower bound is given by the solution of the Lagrangian primal.

Note that, even though routing and link scheduling are decided in two separate modules in Figure 5 (i.e., the Lagrangian primal and the optimal link scheduling), the fact that the Lagrangian scheme iterates between the primal and dual, computing bounds on the activation variables, implies that routing decisions are affected by scheduling decisions and vice-versa, which makes the approach joint in all respects.

A solution approach like this belongs to the Lagrangian heuristics family ([31]). As far as solution efficiency is concerned, we solve the Lagrangian dual via a bundle type method ([29]-[30]), which is more efficient than a standard subgradient method, as it takes into account information from previous iterations when searching for the ascent direction and step.

\section{IV.PERFORMANCE EVALUATION}

The contribution of this section is twofold. First, we evalu- ate the performance of our heuristic approach to solve the DARS problem, in terms of optimality and complexity. Second, we exploit it to infer structural properties of the WMN, i.e. optimal placement of one or more Internet gateway nodes. We present the above contributions in two separate subsections.

\section{A. Evaluation of the heuristic approach}

As for the first objective, we make simulations on a grid of varying diameter, up to $7 \times 7$ nodes. All links have a capacity equal to 9600 , and the gateway is located in one corner. We assume that each link interferes only with those that are one hop away, and set the conflict graph accordingly. One flow is originated at each node, and is to be routed to the gateway. Instances are solved using an Intel Core 2 Duo CPU, $2.33 \mathrm{GHz}$ using IBM ILOG CPLEX 12.1

As for optimality, we compare the optimal DARS solutions, where available (up to a $4 \times 4$ grid) and those computed with the heuristic $L C S+r-D A R S$. In this last approach, the rDARS is solved both optimally and via the Lagrangian heuristic. For each test set, we evaluate the objective on a set of 30 randomly generated instances, with heterogeneous flow requirements: rates and bursts are generated uniformly between $[0,9600 /(2 \cdot|Q|)]$ and $[0,1000]$, while the deadlines are set to either 60 or 90 . Frames have 100 slots. We first show that separating the LCS and the r-DARS yields accurate results. Figure 6 shows the relative gap with respect to the DARS optimum in a $4 \times 4$ grid. The figure clearly shows that the suboptimal solutions of the two schemes are within few percentage points to the optimum.

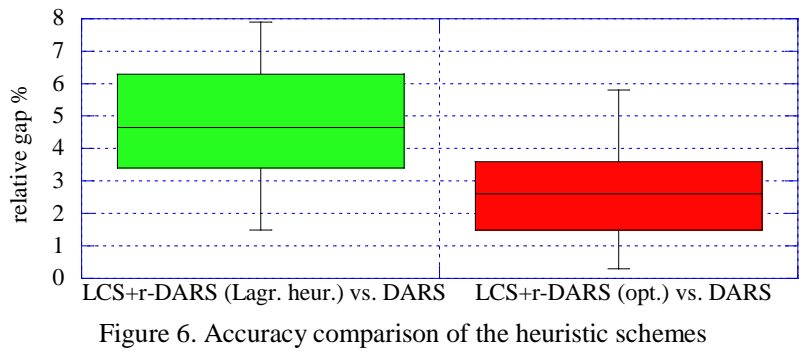

However, solving the r-DARS optimally is time consuming: already with $5 \times 5$ grids, we could not find instances this took less than 8000s. Instead, the Lagrangian heuristic is considerably faster. Figure 7 reports a box plot of the solution times of 30 instances of grids, from $4 \times 4$ to $7 \times 7$. The figure shows that routing plans can be done in a few hours for grids up to $7 \times 7$, which is quite a large dimension for a WMN.

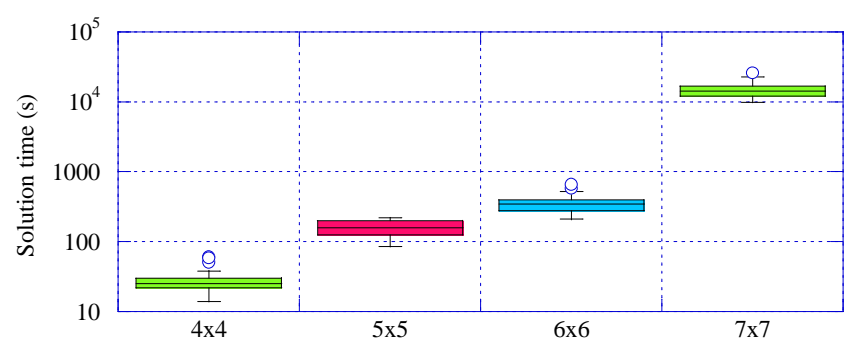

Figure 7. Solution time for the LCS+r-DARS, using the Lagrangian heuristic

Next, we show the benefits of having a joint routing and scheduling, by comparing it to a cascading approach, where routing decisions are taken first, oblivious of link scheduling. 
In the latter, we use a capacitated multicommodity flow (CMF) routing, where each flow $q$ requires a capacity equal to its rate $\rho_{q}$, and the routing that minimizes the overall number of traversed links is chosen, keeping into account the capacity constraints. The CMF sets the $t_{e}^{q}$ variables, and then the link scheduling is solved optimally given the routing, as in [9]. In the joint approach, we use LCS+r-DARS, with the latter solved through the Lagrangian heuristic. Figure 8 shows the relative gap between the cascading and the joint approaches for two sets of instances of a $6 \times 6$ grid: for the first set rates and burst are again generated uniformly between $[0,9600 /(2 \cdot|Q|)]$ and $[0,1000]$, for the second one the rates are generated between $[0,9600 /(1.2 \cdot|Q|)]$; this leads to instances where the WMN is highly congested, with the links close to the gateway approaching the saturation point. For the first set a joint approach (although solved suboptimally) always performs $10 \%-15 \%$ better in terms of objective function, despite the fact that both subproblems are solved optimally in the cascading approach. For the second set the gap grows to $20 \%$. However, the cascading approach fails to compute a feasible solution in as many as $37 \%$ of the instances, whereas our joint approach solves them all.

Then, we show how schedulability of a set of flows changes with their rate and burst. Figures 9-11 show the maximum violation as a function of the burst and rate of the flows. Figures 9 and 10 show results for a burst value of 1000 against a rate from 50 to 300 on a $5 \times 5$ and $6 \times 6$ grid respectively. Figure 11 reports results for a burst size ranging from 0 to 2000 and a rate of 150. In the above figures, the (unfeasible) solution of the continuous relaxation of the r-DARS problem is shown for comparison. The latter is a lower bound on the optimum, and its purpose its to show that - despite we cannot compute the optimum DARS solution - both the r-DARS optimum and its heuristic approximation are quite close to the DARS lower bound, hence to the DARS optimum itself. Note that in the continuous relaxation routing variables are not integer. In this case, constraints (ii-vi) in the DARS model have no physical counterparts.

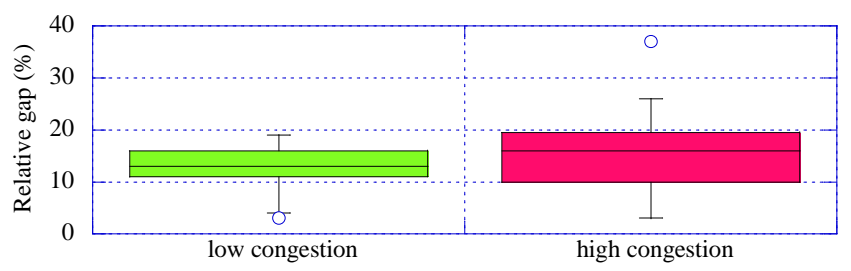

Figure 8 . Relative gap between the cascading and the joint approach (the latter solved through the Lagrangian heuristic) on a $6 \times 6$ grid WMN

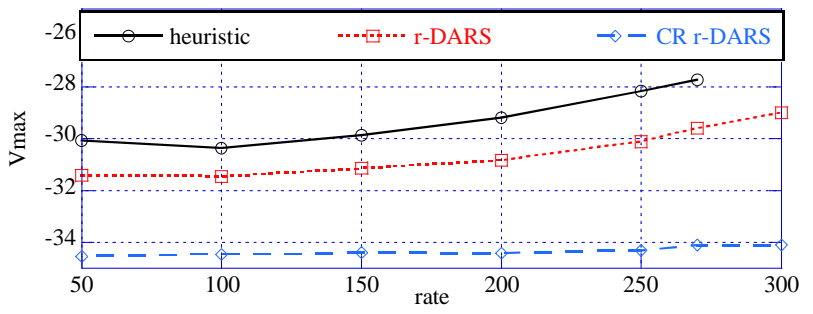

Figure9. Maximum violation as a function of the rate for a $5 \times 5$ grid topology

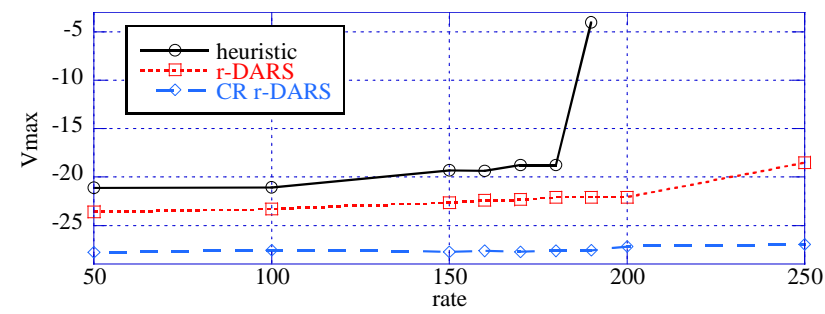

Figure 10. Maximum violation as a function of the rate for a $6 \times 6$ grid topology

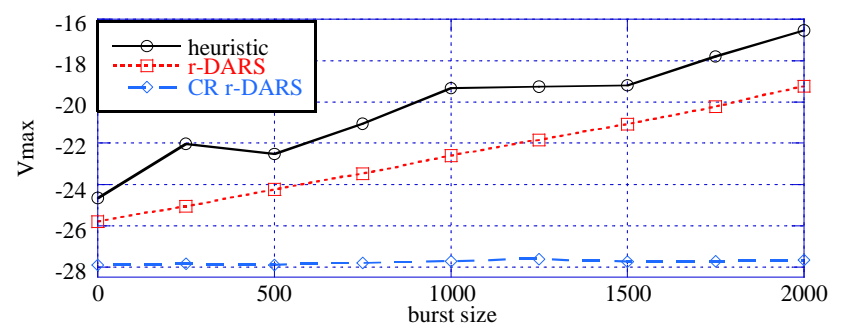

Figure 11. Maximum violation as a function of the burst size

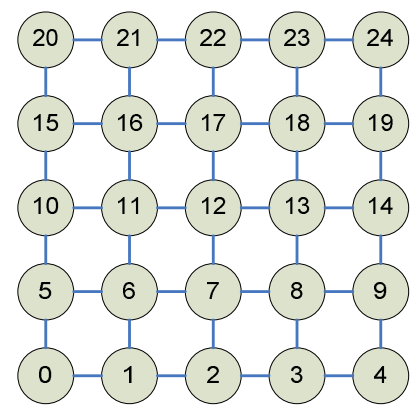

Figure 12. The test-case $5 \times 5 \mathrm{WMN}$

\section{B. Case study: optimal gateway placement}

We now show how to exploit our solution scheme to infer properties which are useful from a network engineer perspective. More specifically, we discuss optimal gateway placement in both single-gateway and multi-gateway WMNs. We take as an example a $5 \times 5$ grid mesh, shown in Figure 12, and we initially place a single gateway and homogeneous traffic, one flow from each node to the gateway. For obvious reasons of symmetry, we only move the gateway toward one border and corner of the WMN. Figure 13 shows $V_{\max }$ as a function of the rate when a single gateway is placed at various nodes, from the center to the corner, for a burst equal to 1000 and a deadline of 60 . The figure shows that $V_{\max }$ is minimized when the gateway lies in the center. The result makes sense since a central gateway minimizes the length of the longest path as well, which are the ones likely to contribute to $V_{\max }$. Figure 14 further clarifies that a larger $V_{\max }$ is obtained at the price of a higher resource expenditure, its vertical axis reporting the sum of the allocated capacity on all the slots of the schedule. Note that it is not possible to obtain a feasible schedule with $\rho=350$ when the gateway is placed in the corner.

We repeated the evaluation with random flows, whose parameters are the same as in the previous section. The results, shown in Figure 15, show that the distribution of $V_{\max }$ moves to the right as we move the gateway from the centre to one corner.

Finally, we compared the single-gateway scenario to one where the WMN has two gateway nodes. Figure 16 shows 
both $V_{\max }$ (left vertical axis) and the allocated capacity (right vertical axis) as a function of the placement of the gateways. The most favorable single-gateway scenario is reported on the left for comparison. All data are related to a homogeneous traffic scenario, with one flow from each non-gateway node whose characteristics are $\rho=100, \sigma=1000$ and $\delta=90$. Note that the two-gateway scenarios have one flow less than the single-gateway scenario, as gateways send no traffic themselves. The figure shows that the more far apart the two gateways are, the worse $V_{\max }$ is, and the higher (in general) is the allocated capacity. However, it also shows that the only result that can be achieved by putting two gateways is to improve $V_{\max }$ marginally, at the price of a $27 \%$ increase in the allocated capacity. Within the limit of the considered scenarios, this suggests that a single gateway, placed at the center, is the optimal solution for a WMN of this topology and traffic.

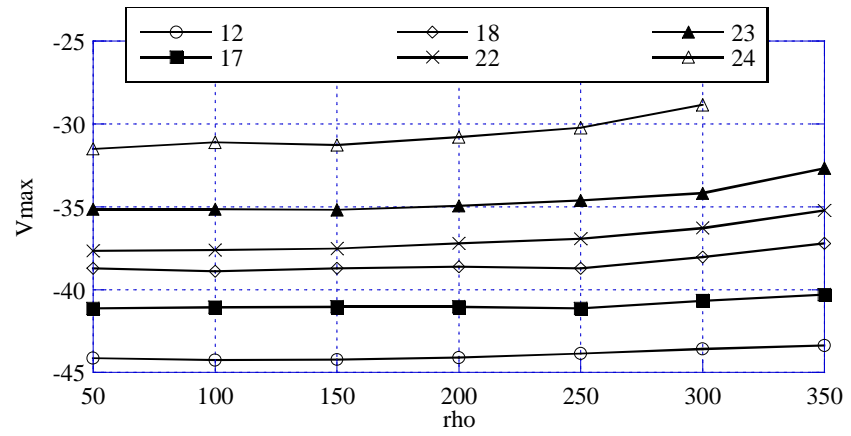

Figure 13 - Vmax as a function of the rate for various gateway placements homogeneous traffic

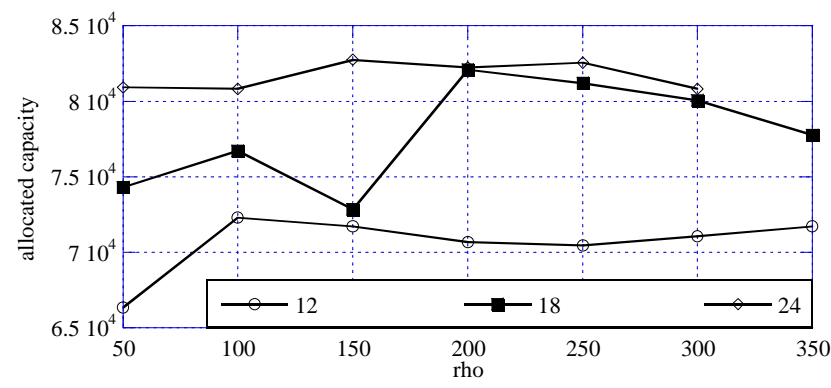

Figure 14 - allocated capacity as a function of the rate for various gateway placements - homogeneous traffic

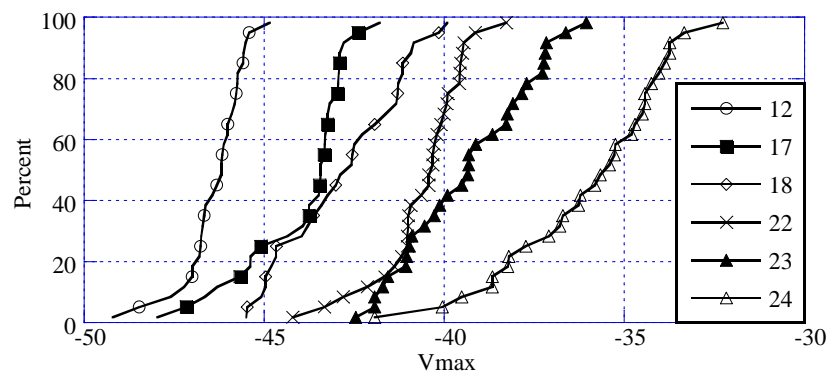

Figure 15 - Distribution of Vmax over 30 random instances with different placements of the gateway node

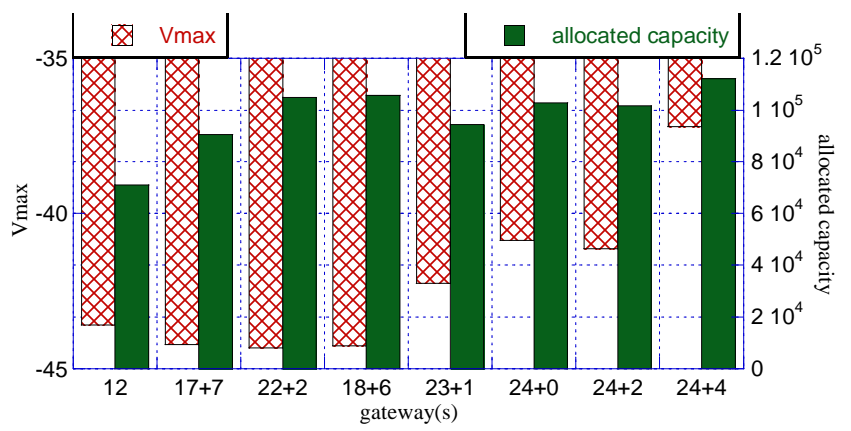

Figure 16 - Vmax and allocated capacity for a single gateway and twogateway scenario

\section{RELATED WORK}

In this section we review some of the related works on routing and link scheduling in WMNs. As the literature on the subject is abundant, we narrow down the scope to those that are more germane to our work, leaving out anything connected with multi-radio systems (where the channel assignment problem is the most prominent issue) and/or not dealing with performance bounds. As already stated, no work that we are aware of (save our previous work on the same topic, [8]-[10]) considered schedulability in WMNs with: i) VBR traffic, and ii) arbitrary end-to-end delay constraints. Most of the link scheduling approaches fall into either of the following categories:

1. rate-oriented algorithms, that either provide flows with a minimum guaranteed rate (e.g. [15]-[18]), or optimize the total throughput (e.g. [19]-[22]). Guaranteeing a minimum rate no smaller than the flow's rate - e.g. by (5) - is a necessary condition for end-to-end delays to be finite, but does not automatically make them smaller than a prespecified bound. In fact, by renouncing over-allocating rates, these schemes often compute schedules with unfeasibly large delays.

2. TDMA delay-oriented algorithms, that either minimize (e.g. [25]-[26]) or try to guarantee a maximum TDMA delay (e.g. [23]-[24]). The latter is the sum of TDMA waiting times at every hop, i.e. the time it takes for a packet to travel from the source to the destination, assuming that it is never queued behind other packets. As queuing is a component (and often the dominant one) of the end-toend delay, especially with VBR traffic, there is no guarantee that such algorithms can actually find a delay-feasible schedule if there exists one. We showed this in [9], using [26] as a comparison.

Within the second category, [25] considers both CBR (voice) and VBR (video) flows, however assuming that VBR sources can be described as stationary, ergodic and independent processes with known statistics, so as to characterize them as equivalent CBR sources. In this work, we deliberately omit this kind of assumptions, sticking instead to more practical $\sigma, \rho$ characterizations, which can be conveyed to the network using standard signaling protocols such as RSVP).

Some works not falling into either of the above categories are also relevant, as they provide frameworks for computing delay bounds a posteriori, after routing and link scheduling have been planned. In [27] authors define the odd/even link ac- 
tivation and routing framework, and employ internal scheduling policies at each link so that the end-to-end delay bound along a path is roughly double than the one obtained in a wired network of the same topology. Authors of [28] show that using throughput-optimal link scheduling and Coordinated-EDF to schedule packets within each link, rate-proportional delay bounds with small additive constants are achieved. Our goal is instead to have pre-specified, arbitrary delay bounds respected through link scheduling.

Finally, some works (e.g., [37]-[38]) consider placing one or more gateways subject to QoS constraint. However, they use additive, per-link delay bounds in their computation, which hold regardless of the traffic traversing them.

\section{CONCLUSIONS AND FUTURE WORK}

In this work we have analyzed Delay-Aware Routing and Scheduling (DARS) problem for WMNs. We have formulated the problem as an optimization problem, which is however too complex to solve optimally already at relatively small scales (e.g., a 4x4 grid WMN). We have devised a heuristic, based on i) extrapolating the link conflict serialization from the rest of the DARS problem, and ii) solving the reduced DARS problem using a Lagrangian heuristic, which allows one to reap the benefits of a joint routing and scheduling approach, without paying the price of the added model complexity. Our results show that the heuristic scheme is fast and accurate, allowing a network administrator to provision a WMN of several tens of nodes so as to meet pre-specified delay guarantees for real-time traffic. Furthermore, we have used the above technique to identify guidelines for the optimal placing of gateways in the WMN.

This is the first work considering delay bounds as an objective, despite the abundant literature on joint routing and scheduling. Future work, which is actively being pursued at the time of writing, will include evaluating our scheme in different settings, i.e. random topologies, and/or using different interference models. In fact, the solving times are directly connected with the degree distribution in the conflict graph, which in turn depends on the topology [39]. Furthermore, we are considering multipath routing, i.e. allowing a traffic flow to be split among several paths in order to balance the link utilization.

\section{REFERENCES}

[1] I. F. Akyildiz, X. Wang, W. Wang: "Wireless mesh networks: a survey", Elsevier Computer Networks, 47:(4), pp.445-487, 15 March 2005.

[2] R. Nelson, L. Kleinrock. "Spatial TDMA: A collision-free multihop channel access protocol", IEEE Transactions on Communications, 33(9), pp. 934-944, 1985.

[3] M. Alicherry, R. Bhatia, L.E. Li. "Joint channel assignment and routing for throughput optimization in multiradio wireless mesh networks", IEEE JSAC, 24(11), pp. 1960-1971, 2006.

[4] M.S. Kodialam, T. Nandagopal. "Characterizing achievable rates in multi-hop wireless networks: the joint routing and scheduling problem", in Proc. ACM MOBICOM'03, San Diego, CA, USA, pp. 42-54, 2003.

[5] V. S. Anil Kumar et al. "Algorithmic aspects of capacity in wireless networks", SIGMETRICS Performance Evaluation Review, 33(1), pp. 133-144, 2005.

[6] T. Moscibroda, R. Wattenhofer. "Coloring unstructured radio networks", in Proc. ACM SPAA'05, New York, NY, USA, pp. 39-48, 2005.
[7] S. Ramanathan. "A unified framework and algorithm for channel assignment in wireless networks", Wireless Networks, 5(2), pp. 81-94, 1999.

[8] P. Cappanera, L. Lenzini, A. Lori, G. Stea, G. Vaglini, "Link Scheduling with End-to-end Delay Constraints in Wireless Mesh Networks", in Proc. of IEEE WoWMoM 2009, Kos, Greece, June 15-19, 2009.

[9] P. Cappanera, L. Lenzini, A. Lori, G. Stea, G. Vaglini, "Optimal Link Scheduling for Real-time Traffic in Wireless Mesh Networks in both Per-flow and Per-path Frameworks", in Proc. WoWMoM 2010, Montreal, CA, June 2010

[10] P. Cappanera, L. Lenzini, A. Lori, G. Stea, G. Vaglini, "Efficient Link Scheduling for Online Admission Control of Real-time Traffic in Wireless Mesh Networks", Elsevier Computer Communications, to appear.

[11] J.-Y. Le Boudec and P. Thiran. Network Calculus. Springer Verlag, LNCS 2050, New York, NY, 2001.

[12] P. Gupta, P.R. Kumar. "The capacity of wireless networks", IEEE Transactions on Information Theory, 46(2), pp. 388-404, 2000.

[13] ILOG CPLEX software, http://www.ilog.com

[14] P. Bonami and J. Lee, BONMIN Users' Manual: see http://projects.coinor.org/Bonmin

[15] R. Bhatia M. Kodialam, "On Power Efficient Communication over Multi-hop Wireless Networks: Joint Routing, Scheduling and Power Control", pp 1457-1466, 2004

[16] J. Kazemitabar, V. Tabatabaee, H. Jafarkhani "Global Optimal Routing, Scheduling and Power Control for Multi-hop Wireless Networks with Interference", 2008

[17] R. Cruz and A. Sanathanam, "Optimal Routing, Link Scheduling and Power Control in Multi-hop Wireless Networks," Proceedings of IEEE INFOCOM 2003.

[18] J. El-Najjar, C. Assi, B. Jaumard, "Joint Routing and Scheduling in WiMAX-based mesh networks: A Column Generation Approach", proc. WoWMoM 2009, Kos, Greece, June 2009

[19] Y. Wang, W. Wang, X.-Y. Li, W.-Z. Song, "Interference-Aware Joint Routing and TDMA Link Scheduling for Static Wireless Networks, IEEE Trans. on Parallel and Distributed Systems, 19(12): 1709-1725, Dec. 2008

[20] J. Crichigno, M. Y. Wu , J. Khoury, W. Shu, “A Joint Routing and Scheduling Scheme for Wireless Networks with Multi-packet Reception and Directional Antennas", proc. WOWMOM 2009, Kos, Greece, June 2009

[21] K. Jain, J. Padhye, V. Padmanabhan, L. Qiu. Impact of interference on multi-hop wireless network performance, proc. ACM Mobicom 2003

[22] O. Goussevskaia, R. Wattenhofer, M. M. Halldorsson, E. Welzl, "Capacity of Arbitrary Wireless Networks", proc. INFOCOM 2009

[23] M. Leoncini, P. Santi, P. Valente "An STDMA-Based Framework for QoS Provisioning in Wireless Mesh Networks", proc. MASS 2008

[24] J. Zou, D. Zhao "Connection-Based Scheduling for Supporting RealTime Traffic in Wireless Mesh Networks", IEEE Trans. on Wireless Communications, 8(3):1182-1187, 2009

[25] H. Shetiya, V. Sharma: "Algorithms for Routing and Centralized Scheduling to Provide QoS in IEEE 802.16 Mesh Networks", in Proc. WMuNeP'05, Montreal, CA, Oct. 13, 2005

[26] P. Djukic, S. Valaee. "TDMA delay aware link scheduling for multi-hop wireless networks", IEEE/ACM Trans. on Networking, 17(3), pp.870883,2009

[27] G. Narlikar, G. Wilfong, L. Zhang, "Designing Multihop Wireless Backhaul Networks with Delay Guarantees", Springer Wireless Networks, August 2008

[28] P. Jayachandran, M. Andrews, "Minimizing end-to-end Delay in Wireless Networks Using a Coordinated EDF Schedule", INFOCOM 2010

[29] A. Frangioni "Generalized Bundle Methods" SIAM Journal on Optimization 13(1), p. 117 - 156, 2002

[30] A. Frangioni, G. Gallo "A Bundle Type Dual-Ascent Approach to Linear Multicommodity Min Cost Flow Problems" INFORMS Journal On Computing 11(4), p. 370 - 393, 1999

[31] C. Barnhart. Dual Ascent Methods for Large-Scale Multicommodity Flow Problems. Naval Research Logistics, 40, p. 305-324, 1993. 
[32] B. Gendrou, A. Herz, P. S. Louis, "On edge orienting methods for graph coloring”, J. Comb. Optim., 13(2) 2007, pp. 163-178

[33] H. Li, Y. Cheng, C. Zhou, W. Zhuang, "Minimizing end-to-end delay: a novel routing metric for multi-radio wireless mesh networks", Infocom 2009, 46-54

[34] M. Cesana, L. Fratta, M. Gerla, E. Giordano, G. Pau, "C-VET the UCLA Campus vehicular testbed: integration of VANET and Mesh networks", European Wireless 2010, Lucca, Italy, April 2010

[35] A. Kandhalu, A. Rowe, R. Rajkumar, C. Huang, C.-C. Yeh, "Real-time video surveillance over IEEE 802.11 mesh networks", RTAS '09, S. Francisco (US) April 13 - 16, 2009

[36] A. Mehrotra, M. A. Trick, "A Column Generation Approach For Graph Coloring”, INFORMS Journal of Computing, 8 (4) 1996, pp. 344-354

[37] Y. Drabu, H. Peyravi, "Gateway Placement with QoS Constraints in Wireless Mesh Networks," ICN, pp.46-51, Seventh International Conference on Networking (ICN 2008), 2008

[38] B. Aoun, R. Boutaba, Y. Iraqi, G. Kenward, "Gateway Placement Optimization in Wireless Mesh Networks With QoS Constraints", IEEE JSAC 24(11):2127-2136, Oct. 2006

[39] D. M. Blough, M.Leoncini, G. Resta, and P. Santi, “The k-Neighbors Approach to Interference Bounded and Symmetric Topology Control in Ad Hoc Networks", IEEE Trans. On Mobile Computing, 5(9): $1267-$ 1282, Sep. 2006 